\title{
DIGITAL DOCUMENTATION OF SHIPS IN CULTURAL HERITAGE: A EUROPEAN REVIEW
}

\author{
A. Colson ${ }^{\text {a* }}$ \\ a German Maritime Museum, Hans-Scharoun-Platz 1; 27568 Bremerhaven, Germany (acn.colson@gmail.com)
}

\section{Commission II}

KEY WORDS: Ships, Digital Documentation, Cultural Heritage, Coordinate Measuring Machine (CMM), Total Station Theodolite (TST), Laser scanning.

\begin{abstract}
:
Ships of different shapes and times are lying in harbours, on land or in museums, all over the world. Our aim with this paper was to review work done on digital documentation of ships in Cultural Heritage based on different initiatives in Europe using Coordinate Measuring Machine (Newport Ship and Doel 1); Total Station Theodolite (Vasa and Mary-Rose) and Laser scanning (LaScanMar and Traditional boats of Ireland). Our results showed that some discrepancy exist between the projects, in terms of techniques and expertise at hand. Furthermore, few guidelines have been in practice but only for Archaeology and Ethnology. However, no standards are existing. Three focuses have emerged: documentation of single ship elements, monitoring of the long-term deformation processes and the documentation of collections of ships. We discussed the diversity of expert's background and the complexity of comparability between projects.

In conclusion, guidelines are necessary to enable a common ground for all professions to work together, e.g. in Architecture. This path must be taken now for digital documentation of ships, if not information and knowledge will be lost on the way.

100-200 words
\end{abstract}




\section{INTRODUCTION}

Maritime Heritage is by essence international and ships have always been the medium of goods and knowledge. Over the last 15 years, projects using different methods flourished all over the world to document digitally historical, archaeological or ethnological ships.

The oversize of these kind of artefacts justifies the use of modern technologies for the documentation. In fact, digital documentation enables on the one hand new assessments that are not possible with traditional methods, but also a higher precision (Jones; 2015).

Several initiatives show the path using coordinate measurement machines, such as a Faro Arm (Jones; 2015) or Total Station Theodolite (Jacobson; 2003a), and Laser Scanners (Tanner; 2012a). Nevertheless, these different initiatives were never reviewed together as a whole, and looked at globally.

Our aim with this paper is to review the current state of digital documentation of ships in Cultural Heritage. 1) identify the discrepancy between different initiatives based on chosen examples coming from Europe; 2) point out the lack of standards and guidelines in the field; 3 ) show the impact of the heterogeneous backgrounds of actors involved.

\section{REVIEW}

\subsection{Coordinate Measuring Machine (CMM)}

\subsubsection{Description}

This instrument was invented in the 1950's and used to control the quality of manufactured pieces in the automotive industry. "Usually [it is] taking the form of articulated mechanical arm, mounted on a solid base, able to rotate on [different] axes". (Ranchin-Dundas; 2012). The CMM is directly linked to a CAD software. The goal is to document single elements digitally and acquire their three-dimensional shape. The details documented and the accuracy relies on the operator, that chooses the relevant information. After post-processing, the 3D data can be used for different purposes.

\subsubsection{Use in Maritime Cultural Heritage}

In Maritime Archaeology, the CMM mostly used has been the one from the company Faro. This instrument was used in the field since 1990's by Mark Starr at the Seaport Museum in Mystic, Connecticut, USA. (Ranchin-Dundas; 2012). In Europe, it was first purchased by the Danish Maritime Museum, in Roskilde in 1999 (Ranchin-Dundas; 2012). Single elements are digitized: the operator touches the object using the highly precise pointer and selects the relevant points. In that manner, tool marks, edges and general shape, as well as cracks or wood grain elements can be documented in separated layers. The information constitutes a base used by the archaeologists for models or scientific reconstructions.

First used in Denmark, it became standard practice in Archaeology to document ship timbers. (Falck; 2013a).

\subsubsection{Examples}

In 2002 , a $15^{\text {th }}$-century ship of about $25 \mathrm{~m}$ of length was found in the River Usk, close to the Severn Estuary on the western seaboard of Britain, in Newport, Wales (Nayling; 2012a).

The Newport Ship Project, used CMM Faro Arm to document all the ship timbers after the recovery and became the reference (Ranchin-Dundas; 2012). The documentation of over 3,000 timbers and fragments, was made over 2 years, with four instruments running parallel. (Jones; 2015). After the conversion of all timbers data into solids and 3D printing at $1: 10$ scale, the ship has been physically reconstructed. (Jones; 2013). Later on, the model of the ship was scanned and the data processed as a polygon mesh surface. At that stage, the missing parts of the ship and its theoretical behaviour in the water called hydrostatic data were added. Thanks to the software Orca 3D (Tanner; 2013b) a digitally optimized model was produced getting closer to the original ship.

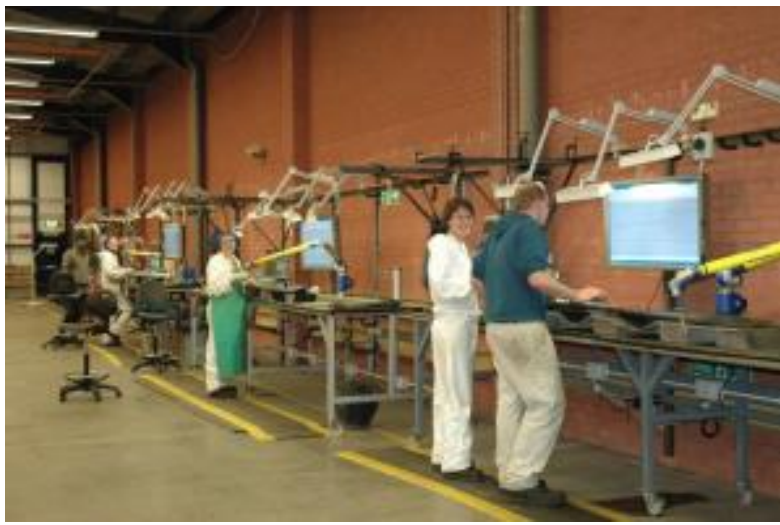

Figure 1. Four Documentation stations (CNewport Museums and Heritage Service

Close to Antwerp, Belgium, a c. $21 \mathrm{~m}$-long ship was found in 2000 during enlargement works of the harbour. The $14^{\text {th }}$. century ship had to wait dismantled in water tanks until 2009 for its documentation (Lenaerts; 2014a). A multidisciplinary team worked on the digital documentation and analysed the remains of the Doel 1 (Vermeersch; 2015a). The documentation was made with a Faro Arm, following the guidelines provided by Toby Jones established at the Newport Ship project (Jones; 2013) and a 1:10 scale model was 3D printed.

In both case, the focus was made on the archaeological data or information linked to it, that would be useful for further research.

\subsubsection{Advantage/Disadvantages}

The investment costs for purchasing a CMM are relatively high and additional software like Rhinoceros 3D and Orca3D is necessary to produce highly valuable results. Nevertheless, this method enables simultaneously to capture spatial information and add the expert's interpretation (Nayling; 2012a).

A training is mandatory to use the instrument for the user to become fully operational. Double checks are necessary by the person in charge if the documentation is not done by the expert.

Guidelines have been established in Newport and a network has been founded: Faro Rhinoceros Archaeological Users Group (FRAUG). In 2017, the yearly meeting took place at the German Maritime Museum in Bremerhaven.

By now, several institutes in Europe own such an instrument.

\subsection{Total Station Theodolite (TST)}

\subsubsection{Description}

A total station theodolite is a surveying instrument using laser light. The distance between the instrument and the target is measured and recorded. It is considered a direct surveying technique because the operator chooses the acquired points manually.

The device integrates the functions of a theodolite (transit) for measuring angles, with an electronic distance meter (EDM) 
and a digital recorder. Angles and distances are measured from the total station to points under survey, and the spatial coordinates $(\mathrm{X}, \mathrm{Y}, \mathrm{Z})$ are calculated using trigonometry and triangulation. The final output is a sequence of points with three-dimensional coordinates in relation to a local or geographical reference system (Howard; 2007).

This technique is used on building sites, as well as on archaeological excavations to measure large distances and establish maps (Andrews; 2009).

\subsubsection{Use in Maritime Cultural Heritage}

Since the 1990's TST was used to document archaeological ships but later also for scheduled monitoring. For documentation, it has been often used combined with other methods such as hand drawing and photography. (RanchinDundas; 2012). The operator used a reflective prism as a target for improved accuracy but the method also works reflectorless. Since 2011, the methodology developed by Frederik Hyttel and presented in his Master's thesis, using real-time CAD recording, made documentation on the "fly", faster and easier (Hyttel; 2011).

On the other hand, scheduled monitoring must be careful planed and the procedure repeatable to allow the comparison of different datasets. A few initiatives do exist, but the protocol developed in Sweden was one of the first.

\subsubsection{Examples}

A TST was used at the Vasa Museum, in Stockholm, Sweden, on the $17^{\text {th }}$-century ship. In that case, the aim was to settle a long-term measuring system to monitor the change of shape. In the late 1990's, the experts were concerned about preserving the ship's shape over time and it was decided to look for a solution to acquire the geometry precisely and compare the data over the years. The partnership with a Royal Institute of Technology (KTH) in Stockholm allowed Dr. Milan Horemuz coming from Geo-Information Technology and Geodesy to work as a consultant on a monitoring protocol. Since 2000, two acquisitions per year are undertaken by the museum's staff with 301 reflective targets from 66 different positions on the outside and extended with 69 prisms on the inside (van Dijk; 2016a). Since 2003, a digital visualisation of the result is available programmed on MATLAB (Jacobson, 2003a). Each acquisition requires 20 man-days. The distance between points can be compared and cross sections selected to investigate deformation.

In Portsmouth, Great-Britain, the Mary-Rose, King Henry VIII's ship from the $16^{\text {th }}$-century, was the subject of investigation concerning her shape. Since 2013, the ship has been drying slowly after a long conservation treatment with Polyethylene Glycol. During that phase, the construction is very fragile and deformations can occur. Therefore, certain measures were taken and a Geodetic solution was favoured. The focus is made on the ship's extremities that have been identified previously by the team as critical and 31 points are measured constantly (Schofield; 2016a).

Both initiatives were concentrating on the long-term preservation aspects and conservation of the ships. At the time of writing this article, both projects were still on-going.

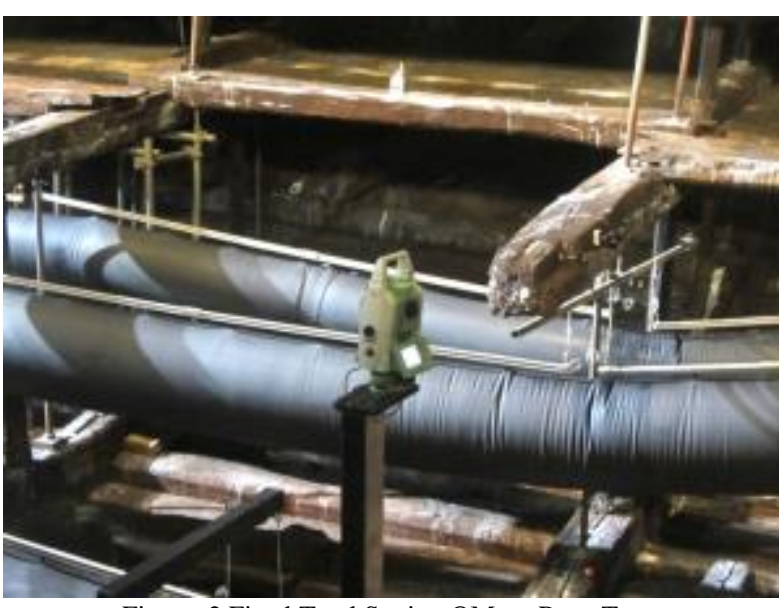

Figure. 2 Fixed Total Station @Mary Rose Trust

\subsubsection{Advantage/Disadvantages}

In both examples, the solution has been exclusively designed for the ship and serves the conservation need. The instrument is rather common in the field of archaeology and the price is not an obstacle.

On the other hand, the setup is relatively complex for monitoring and must be develop by experts from the field. In both cases, external expertise, not at hand in the museum, has been necessary to design a robust acquisition protocol. Nevertheless, the people in charge still report the difficulty of analysing the data and the lack of automatized solution to visualize the deformation.

\subsection{Laser Scanning}

\subsubsection{Description}

The scanner measures distances between the machine and the target using a laser light. Placed on a tripod, similar to the total station, the laser is launched and the time necessary to come back from the target indicates the distance (Howard; 2007). The machine scans systematically its surroundings and establishes a point cloud containing all geo-information. Depending on the kind of scanner, surface information can be acquired additionally, providing colour. (Andrews; 2009)

\subsubsection{Use in Maritime Cultural Heritage}

Numerous projects were using Laser Scanning for documentation of ships of different sizes. Since the mid 2000's, institutes having the opportunity to scan their masterpiece took the chance applying for funding.

Often, the scanners are purchased for a specific project and the staff is trained. In some other cases, a company or even the producers get involved to participate to case studies.

The most interesting projects deal with series of scan, where the procedure and the data processing is thought through. Documentation is the focus, but the amount of data having to be produce with the same format, is always the main challenge.

\subsubsection{Examples}

The Project LaScanMar (Laser Scanning Maritime) was financed by the European Regional Development Fund from the European Union. The Port Musée, Douarnenez (France) and National Museum Cornwall (England) decided to "develop a common scanning method [to acquire] (...) the boat collections". The aim was to "preserve traditional boats from degradation and contribute to their conservation. Promote the common maritime heritage (...) by backing up 
data and 3D digital reconstructions of boats." (Interreg4amanche; 2014).

From 2012 to 2014, with a budget of ca. 140,000€, all together 20 boats were digitised with a laser scanner and the information was made accessible to the public on their website (LaScanMar; 2017).

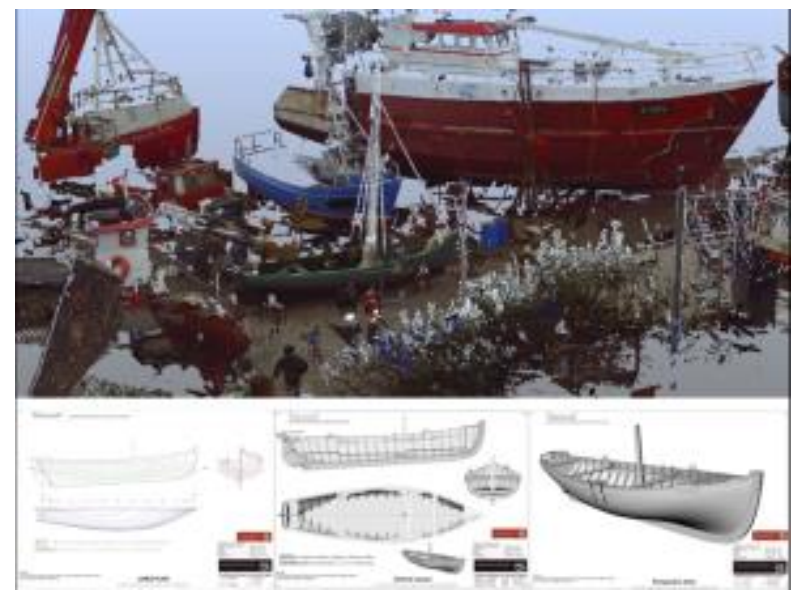

Figure 3. Laser Scanning - Traditional Boats of Ireland @Pat Tanner

The "Traditional Boats of Ireland" project was an independently-funded initiative holding the support of the Heritage Council of Ireland. The aim was a comprehensive survey of Ireland's traditional water craft using laser scanning technology. Since 2008, digital recording of scale or presentation model, builders' half models and builders' framed models has been undertaken. For each boat, a two-dimensional line plan is produced together with hydrostatic data. The scanning process was developed by Pat Tanner, when he joined the project in 2009 and his background in boatbuilding, Architecture and CAD appeared to be very useful. (Tanner; 2012a).

By now, ca. 30 boats have been digitally recorded. At the time of writing this article, the project was still on-going.

Both projects developed an 3D acquisition protocol to document a series of boats, which reveals an ethnological mind-set.

\subsubsection{Advantage/Disadvantages}

The data acquired is known for its accuracy and used in building industry as well as in cultural heritage. The amount of data produced is large and the processing must be made by experts, moreover when the acquisition is composed from several scans on different positions. Although different price classes emerged on the market the last 5 to 10 years, it remains an expensive tool (Howard; 2007).

Operating a laser scanner demands at least a training in the surveying field or even an adequate education.

Nevertheless, after post-processing, a laser scan containing colour information can be used as substrate for communication with the public.

\section{DISCUSSION}

After a short review of different projects and initiatives in the field of digital documentation of ships in Cultural Heritage in Europe, the discussion is focussing on three aspects: 1) Data sets and technologies; 2) Technical background and 3) Framework.

\subsection{Data sets / technologies}

By now, the variety of technologies and datasets is a proven fact. Different technologies and methodologies were used, but a certain sense behind every chosen documentation can be identified.

In the case of the coordinate measuring machine, in both examples a Faro-Arm, the documentation is serving Archaeological interests. For the laser scanning, the goal is to document a series of similar objects, where the data must be comparable and leave as much information possible for posterity, serving an ethnological mind-set. The two examples, using a Total Station Theodolite on the last hand, focus on the long-term preservation and the conservation of two ships for future generation.

In other words, different goals and/or mind-set uses digital technologies for the documentation of ships. As a result, data sets are heterogeneous and every single initiative is unique. The uniqueness of every case makes the comparison of data a challenge.

\subsection{Technical Background}

Not only are the technologies used for the documentation diverse, but also the background of the operators. The experience and the expertise of the person in charge are of defining the documentation is revealed by the results obtained. One would document what she/he knows, which makes methodologies "operator dependent", an advantage and a disadvantage. The information documented is the one, which matters from her/his perspective.

At present, the education of 3D methods applied to Cultural Heritage is not part of all curriculums in the Humanities. This situation leads to a lack of expertise and forces professionals to learn by themselves or to maintain old methods. On the other hand, engineers are asked to participate and join projects with the mind-sets of their field. Interdisciplinarity is often presented only as positive, when obstacles still stand in the way and must be taken into consideration (Brewer, 1999a).

\subsection{Framework}

As of now, Toby Jones' guidelines give a framework for the documentation of ship timbers in archaeology using Coordinate Measuring Machine. (Jones; 2013)

On the other hand, the Heritage council guidelines for surveying heritage boats defines the work done on ethnological boats (Tanner; 2017).

In fact, the scientific literature is poor concerning digital documentation guidelines but full of different case study. Every discipline having a need for digital documentation works on its own and tests different technologies. Anyhow, no standards or general framework for the documentation of ships in Cultural Heritage is formulated.

However, every project claims to make data available to other disciplines for further work, using data collected for other purposes remains tricky.

\section{CONCLUSION}

The review of current state of practices shows that digital documentation of ships in Cultural Heritage is done with various techniques such as Coordinate Measuring Machines, Total Station Theodolite and Laser Scanning. The data produced is very heterogeneous and doesn't enable systematic comparison. The documented elements and the aim vary, which prevents the possibility of a universal dataset. 
Digital documentation of ships in Cultural Heritage opens clearly a new field of investigation. The use of software to test hydrostatic behaviours on the water or simulate future deformation is promising.

We like to think that the data collected is usable in other context by other disciplines. However, given the variety of methodology applied as well as mind-sets, is this goal achievable? Is it realistic to believe that everyone gets what she/he needs if no dialogue exists?

By now, every initiative has been conducted and influenced in each case by a different discipline, which reduces its global impact on digital documentation of ships. We should be looking at the issue from a broader distance and realise that guidelines and standards are necessary to structure the knowledge collected. If not, information will be left aside and never recovered.

In other multidisciplinary disciplines involving experts from different professions, e.g. in Architecture, guidelines have been developed to provide a common ground for effective discussion. A universal framework for the digital documentation of ships in Cultural Heritage, and not specific to archaeology, ethnology or conservation, should be taken into consideration.

\section{ACKNOWLEDGEMENTS}

The support of the COST-Action TD1201 Colour and Space in Cultural Heritage (COSCH) both financial and intellectual, was the first millstone of this work.

The German Maritime Museum supports this research, thanks to Sunhild Kleingärtner and Ursula Warnke, the Museum Directors, that both believed in the project.

A special thanks to the open-mindedness of Kristofer Gamstedt, the enthusiasm and proof-reading of Nico van Dijk (Uppsala University, Sweden). During our discussion, the relevance of the review became a self-evident fact.

Many thanks to Toby Jones (Newport Ship Project, Wales, Great-Britain) for long discussions around this topic.

And finally, thanks to Eleanor Schofield (Mary Rose Trust, Portsmouth, Great-Britain) and Pat Tanner (Traditional boats of Ireland, Baltimore) for kindly providing me nice figures.

\section{REFERENCES}

Andrews, D., Blake, B., Bryan, P., Cromwell, T., and Lea, R., 2009. Measured and Drawn: Techniques and Practice for the Metric Survey of Historic Buildings. Edited by Jon Bedford and Heather Papworth. $2^{\text {nd }}$ Edition. English Heritage.

Brewer, Garry D., 1999a. The challenges of interdisciplinarity. Policy Sciences, 32, pp 327-337.

Falck, T., Egenberg, I.M., Vangstad, H., 2013a. Digital Documentation for Many Purposes: The Barcode 6 Boat as a Case Study. Underwater Archaeology Proceedings, pp 151157.

Howard, P., 2007. Archaeological Surveying and Mapping Recording and Depicting the Landscape. Routledge, London/ New York.

Hyttel, F., 2011. Digital Recording in Maritime Archaeology - Total Station, Rhinoceros and Termite. Master's thesis submitted at the University of Southern Denmark, Esbjerg.
Interreg4a-manche; 2014. Project report - https://interreg4amanche.normandie.fr/en/node/38

Jacobson, J., 2003a. Measuring the Vasa. The Built Environment, 65, pp. 183-190.

Jones, T., Nayling, N., and Tanner, P., 2013a. Digitally Reconstructing the Newport Medieval Ship: 3D Designs and Dynamic Visualisations for Recreating the Original Hull, Form, Loading Factors, Displacement and Sailing Characteristics. Underwater Archaeology Proceedings, pp. 123-129.

Jones, T. 2013. "The Newport Medieval Ship - Timber Recording Manual.", Newport, Wales, Great-Britain. http://archaeologydataservice.ac.uk/archiveDS/archiveDownl oad? $\mathrm{t}=$ arch $-1563-$

1/dissemination/pdf/Newport_Medieval_Ship_Project_Timbe r_Recording_Manual.pdf

Jones, T., 2015. Three-Dimensional Digital Recording and Modelling Methodologies for Documentation and Reconstruction of the Newport Medieval Ship. PhD Thesis submitted at University of Wales Trinity Saint David, GreatBritain.

LaScanMar, 2017. Online data, for the public http://www.lascanmar.com/boats/

Lenaerts, T., 2014a. The Doel Ships: Research aims approaches in methodology. IKUWA V, pp 619-629.

Nayling, N. and Jones, T., 2012a. Three-Dimensional Recording and Hull Form Modelling of the Newport (Wales) Medieval Ship. Proceedings of the 12th International Symposium on Boat and Ship Archaeology. Istanbul, pp. 319324.

Ranchin-Dundas, N., 2012. Three-dimensional (3D) recording of recovered shipwrecks. Master's Thesis submitted at the University of Southern Denmark, Esbjerg.

Schofield, E., McConnachie, G., and Jones, M., 2016a. Air Drying of the Mary Rose Hull. Proceedings of the 12th ICOM-CC Group on Wet Organic Archaeological Materials Conference, May 2013. Istanbul, pp. 398-402.

Tanner, P., 2012a. 3D Laser Scanning in Boat Recording. Nautical Archaeological Society Newsletter.

Tanner, P., 2013b. 3D Laser Scanning for the Digital Reconstruction and Analysis of a $16^{\text {th }}$-Century Clinker Built Sailing Vessel. Underwater Archaeology Proceedings, pp. 137-149.

Tanner, P., 2017.

http://www.tradboats.ie/publications/guidelines.php

Van Dijk, N., 2016a. Monitoring archaeological wooden structures: Non-contact measurement systems and interpretation as average strain fields. Journal of Cultural Heritage, 17, pp. 102-113. 
Vermeersch, J., Haneca, K., Daly, A., 2015a. Doel 2: a

second 14th-century cog wrecked in den Deurganck, Doel,

Belgium. The International Journal of Nautical Archaeology,

44.2, pp. 327-348.

Revised June 2017 